\title{
A case of ramucirumab-related gastrointestinal perforation in gastric cancer with small bowel metastasis
}

\author{
Shinya Urakawa ${ }^{1}$, Daisuke Sakai ${ }^{1,2^{*}}$, Yasuhiro Miyazaki ${ }^{1}$, Toshihiro Kudo ${ }^{1,2}$, Aya Katou ${ }^{1,2}$, Chiaki Inagaki ${ }^{1,2}$, \\ Koji Tanaka ${ }^{1}$, Tomoki Makino ${ }^{1}$, Tsuyoshi Takahashi ${ }^{1}$, Yukinori Kurokawa ${ }^{1}$, Makoto Yamasaki ${ }^{1}$, Kiyokazu Nakajima ${ }^{1,3}$, \\ Shuji Takiguchi ${ }^{1}$, Taroh Satoh ${ }^{1,2}$, Masaki Mori ${ }^{1}$ and Yuichiro Doki ${ }^{1}$
}

\begin{abstract}
Background: Ramucirumab is a monoclonal antibody targeting vascular endothelial growth factor receptor 2 (VEGFR-2). Ramucirumab either alone or in combination with paclitaxel (PTX) has been found to be safe and effective for patients with previously treated advanced gastric cancer. One of the serious adverse events associated with ramucirumab is gastrointestinal (Gl) perforation.

Case presentation: We report the case of a 67-year-old man who developed a ramucirumab-related Gl perforation while undergoing treatment for gastric cancer with small bowel metastasis. He underwent laparoscopic total gastrectomy following neoadjuvant chemotherapy in January 2015 and was diagnosed with hepatic and bone recurrence in October 2015. Ramucirumab in combination with PTX was administered for one and half months after first-line chemotherapy failure. He presented with abdominal pain 7 days after the last ramucirumab dose, and emergency exploratory surgery revealed a small intestinal perforation. Pathological findings indicated that it occurred in a zone containing a small intestinal tumor, which was found to be metastasis of the gastric cancer. He had no postoperative complications, but chemotherapy was not reintroduced and he died 3 months later.
\end{abstract}

Conclusion: We present a recent case of ramucirumab-related gastrointestinal perforation in gastric cancer with small bowel metastasis. This case is rare, but important to consider.

Keywords: Ramucirumab, Gastrointestinal perforation, Gastric cancer, Small bowel metastasis

\section{Background}

Ramucirumab (Cyramza; IMC-1121B; LY3009806; Lilly Oncology, Lilly Research \& Development, New York, NY, USA) is a monoclonal antibody targeting vascular endothelial growth factor receptor 2 (VEGFR-2) [1]. VEGFR-2 inhibition reduces tumor vascularity and growth in animal models [2]. Ramucirumab alone or in combination with paclitaxel (PTX) was proven safe and effective for administration after first-line chemotherapy of advanced gastric cancer and gastroesophageal

\footnotetext{
* Correspondence: dsakai@cfs.med.osaka-u.ac.jp

'Department of Gastroenterological Surgery, Graduate School of Medicine,

Osaka University, 2-2, E-2, Yamadaoka, Suita, Osaka 565-0871, Japan

${ }^{2}$ Department of Frontier Science for Cancer and Chemotherapy, Osaka

University Graduate School of Medicine, E21-19, 2-2, Yamadaoka, Suita,

Osaka, Japan

Full list of author information is available at the end of the article
}

junction adenocarcinoma [3, 4]. Ramucirumab is known to be associated with hypertension, proteinuria, bleeding, and gastrointestinal (GI) perforation [3-7], a rare but life-threatening and emergent adverse event [8].

Here, we report a case of ramucirumab-related gastrointestinal perforation in gastric cancer with small bowel metastasis.

\section{Case presentation}

In November 2014, a 67-year-old man was referred to our hospital due to gastric cancer with concurrent left adrenal metastasis. PET scan revealed high FDG uptake at the primary tumor (maximum standardized uptake value (SUV-max), 10.2) and at the left adrenal gland (SUV-max, 4.5). He had no prior or family history of cancer. Following combination chemotherapy with 
docetaxel, cisplatin, and $\mathrm{S}-1$, he achieved partial response; moreover, PET-CT showed regression of FDG uptake at the primary tumor (SUV-max, 7.5) and disappeared at the left adrenal. No other metastasis was detected. Secondary laparoscopy revealed no peritoneal dissemination and negative with peritoneal washing cytology. Therefore, he underwent laparoscopic total gastrectomy with D2 lymph node dissection (Roux-en-Y reconstruction) and combined resection of the left adrenal gland in January 2015. The pathological diagnosis was moderately differentiated adenocarcinoma, $40 \times$ $30 \mathrm{~mm}$, ypT4a(SE), ly2, v0, ypN0(0/19), ypM1(ADR), Grade 1a, and HER2-positive.

He was diagnosed with hepatic and bone recurrence during S-1 adjuvant chemotherapy in October 2015 and underwent first-line chemotherapy with cisplatin, S-1, and trastuzumab. After first-line chemotherapy failure, he received two courses of ramucirumab at $8 \mathrm{mg} / \mathrm{kg}$ and PTX at $80 \mathrm{mg} / \mathrm{m}^{2}$ beginning on April 12, 2016. His PS was 0 at the beginning of second-line chemotherapy. May 31, 2016, on the appointment date, 1 week after the last ramucirumab administration, he visited to our hospital with abdominal pain which onset was 3 days ago. A physical examination revealed left lower quadrant pain and rebound tenderness. He had a blood pressure of 125/ $75 \mathrm{mmHg}$, body temperature of $38.2^{\circ} \mathrm{C}$, a white blood count of $4400 / \mathrm{mm}^{3}$, and a C-reactive protein level of 33.63. An abdominal CT-scan showed free air and fluid, but no intestinal obstruction (Fig. 1). Emergency exploratory surgery was performed under the impression of peritonitis, and the surgery revealed a large amount of dirty fluid throughout the abdominal cavity. A small intestinal perforation was identified $50 \mathrm{~cm}$ distal from the site of jejuno-jejunal anastomosis (Roux-en-Y reconstruction) (Fig. 2), and there was no peritoneal dissemination. We performed small bowel segmental resection and functional end-to-end anastomosis. He had no postoperative complications and was transferred to another hospital on postoperative day 23 for radiation for his bone metastasis. He was introduced to palliative care for disease progression following the radiation treatment and died 3 months later.

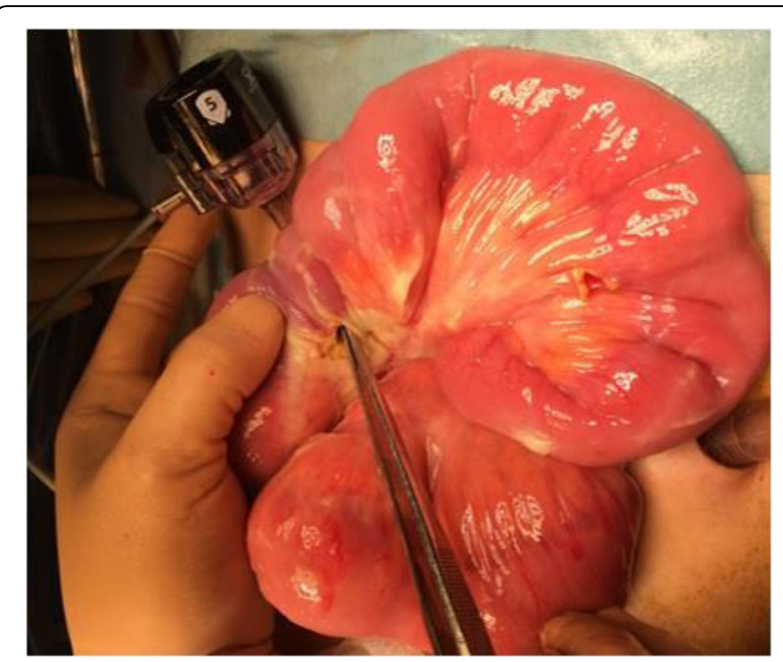

Fig. 2 Intraoperative findings revealed a large amount of dirty fluid in the abdominal cavity. A small bowel perforation was identified $50 \mathrm{~cm}$ distal from the site of jejuno-jejunal anastomosis(Roux-en-Y reconstruction)

The macroscopic findings indicated that the perforation occurred in the resected small bowel segment. No neoplastic lesions were found, but the microscopic findings showed that invasive adenocarcinoma had infiltrated from the submucosa to the subserosa, rather than the epithelial cells. Degeneration and necrosis due to chemotherapy were found in the tumor tissue (Fig. 3).

\section{Discussion}

Gastric cancer is the fourth most common malignant disease and the second leading cause of cancer mortality worldwide [9]. The first-line chemotherapies for unresectable advanced gastric cancer or recurrent gastric cancer are fluoropyrimidine-based and platinum-based combinations [10]. Ramucirumab is a human IgG1 monoclonal antibody VEGFR-2 antagonist [1] and was proven to be effective for advanced gastric cancer. Ramucirumab alone or in combination with PTX prolonged survival in two randomized controlled trials including patients with previously treated advanced

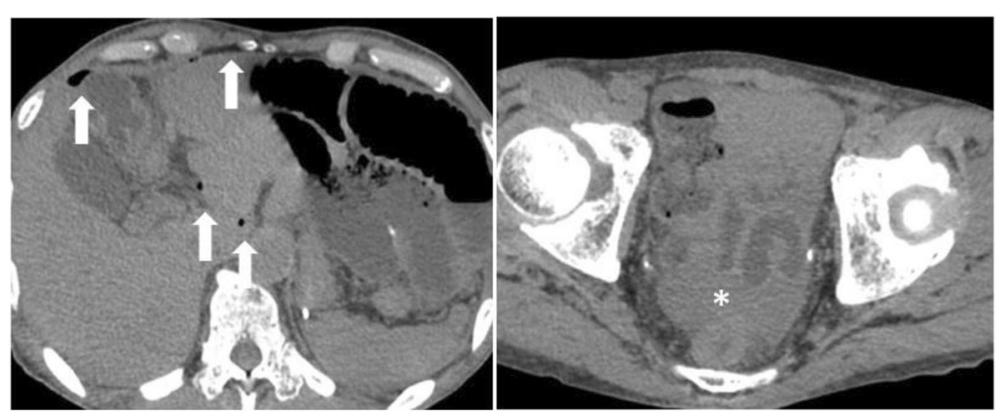

Fig. 1 Free air and fluid in the peritoneal cavity visible on abdominal CT 


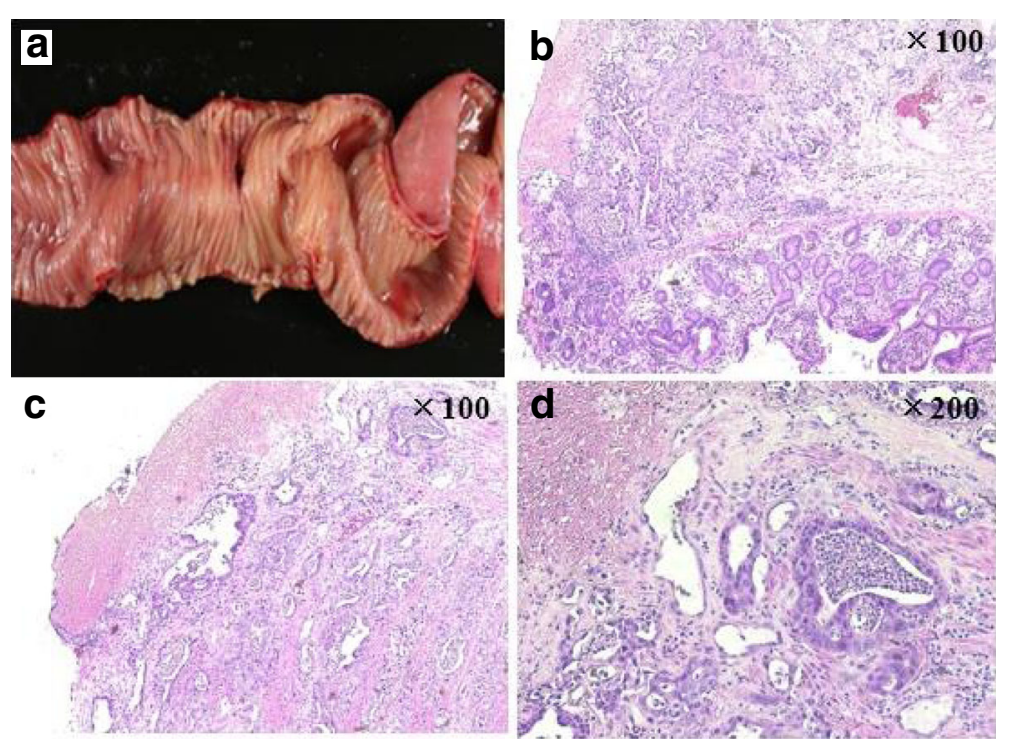

Fig. 3 a-d Macroscopic and microscopic findings showed that Gl perforation occurred in an area of tumor tissue and that invasive adenocarcinoma was found from the submucosa to the subserosa, rather than in the epithelial cells

stomach cancer and gastroesophageal junction adenocarcinoma (REGARD and RAINBOW) [3, 4]. Ramucirumab is generally well tolerated and has acceptable toxicity that includes hypertension, proteinuria, and minor hemorrhage [3-7]. GI perforation is a rare but serious adverse event. The incidence of all-grade GI perforation with ramucirumab was $1.5 \%$ (95\% CI $1.1-2.1 \%$ ) with a mortality of $29.8 \%$ (95\% CI $14.9-50.7 \%$ ) in a previous study [8].

However, the risk factors for GI perforation with ramucirumab have not yet been investigated, and bevacizumab, an anti-angiogenesis inhibitor-like ramucirumab, is also known to increase the risk of GI perforation. Several clinical factors, including peptic ulcer disease, diverticulitis, intestinal obstruction, underlying cancer, recent endoscopy, abdominal radiotherapy, steroid or nonsteroidal anti-inflammatory drug (NSAID) use, and bowel resection at surgery, were considered to increase the risk of GI perforation in patients treated with bevacizumab [11-15]. These risk factors could lead to a recommendation for a different antiangiogenic agent treatment, such as ramucirumab. In the case presented here, the GI perforation was related to a small bowel metastasis.

Gastric cancer metastasis to the small intestine is very rare, and almost all metastases to the small intestine are direct invasions or disseminations, making lymphatic or hematogenous metastases especially rare $(9.1 \%$ of metastatic tumors to the small intestine) [16]. In this case, the tumor in the small intestine was considered a hematogenous metastasis of the gastric cancer because (1) this tumor was histologically the same as the gastric cancer, (2) there was no peritoneal dissemination, and
(3) hematogenous spread is more typical and often includes the adrenal gland, liver, and bone.

The mechanisms of ramucirumab-related GI perforation remain unclear, and the current understanding is based primarily on other anti-angiogenesis inhibitors. One mechanistic hypothesis is tumor necrosis, which would lead to a weakening of the intestinal wall [17]. This hypothesis could account for the perforation reported here, since it occurred in an area of neoplastic infiltration of the small intestine. A second hypothesis is intestinal mesenteric vessel thrombosis or vasoconstriction, which could cause bowel ischemia [18, 19]. We observed these symptoms in macro- and microscopic findings in our case.

Ramucirumab-related GI perforation is difficult to manage since both antiangiogenic agent treatment and the chemotherapy-related immunocompromised condition of the patient impair wound healing. The choice of a conservative or operative approach should be based on the condition of the individual [11], and whether ramucirumab should be reintroduced is uncertain.

It is difficult to detect an intestinal lesion as small as the one presented here, but the best approach to ramucirumab-related gastrointestinal perforation remains surveillance and the prevention of clinical risk factors.

\section{Conclusions}

We present a recent case of ramucirumab-related gastrointestinal perforation in gastric cancer with small bowel metastasis. This case is rare, but important to consider. 


\section{Abbreviations}

Gl: Gastrointestinal; PTX: Paclitaxel; VEGFR-2: Vascular endothelial growth factor receptor 2

\section{Acknowledgements}

DS, TK, and TS received research funding from Yakult Honsha, Chugai Pharmaceutical, and Ono Pharmaceutical. TK received advisory fee from Bayer. TS received lecture fee from Chugai Pharmaceutical, Eli Lilly, Merck-Serono, and Ono Pharmaceutical. The other authors have no conflict of interest or financial ties to disclose. All authors have contributed significantly to this case report.

\section{Authors' contributions}

All authors participated in the management of the patient in this case report. DS supervised the case and the writing of the manuscript. YD is a chairperson of our department and supervised the entire process. All authors read and approved the final manuscript.

\section{Consent for publication}

Written informed consent was obtained from the patient for publication of this case report and the accompanying images. A copy of the written consent form is available for review by the Editor-in-Chief of this journal.

\section{Competing interests}

The authors declare that they have no competing interests.

\section{Publisher's Note}

Springer Nature remains neutral with regard to jurisdictional claims in published maps and institutional affiliations.

\section{Author details}

'Department of Gastroenterological Surgery, Graduate School of Medicine, Osaka University, 2-2, E-2, Yamadaoka, Suita, Osaka 565-0871, Japan. ${ }^{2}$ Department of Frontier Science for Cancer and Chemotherapy, Osaka University Graduate School of Medicine, E21-19, 2-2, Yamadaoka, Suita, Osaka, Japan. ${ }^{3}$ Division of Next Generation Endoscopic Intervention (Project ENGINE), Global Center for Medical Engineering and Informatics, Center of Medical Innovation and Translational Research, Osaka University, 2-2, Yamadaoka, Suita, Osaka 565-0871, Japan.

Received: 5 September 2017 Accepted: 29 November 2017

Published online: 19 December 2017

\section{References}

1. Spratlin JL, Cohen RB, Eadens M, et al. Phase I pharmacologic and biologic study of ramucirumab (IMC-1121B), a fully human immunoglobulin G1 monoclonal antibody targeting the vascular endothelial growth factor receptor-2. J Clin Oncol. 2010;28:780-7.

2. Jung YD, Mansfield PF, Akagi M, et al. Effects of combination anti-vascular endothelial growth factor receptor and anti-epidermal growth factor receptor therapies on the growth of gastric cancer in a nude mouse model. Eur J Cancer. 2002;38:1133-40.

3. Fuchs CS, Tomasek J, Yong CJ, Dumitru F, Passalacqua R, Goswami C, et al. Ramucirumab monotherapy for previously treated advanced gastric or gastro-oesophageal junction adenocarcinoma (REGARD): an international, randomised, multicentre, placebo-controlled, phase 3 trial. Lancet. 2014;383: 31-9.

4. Wilke H, Muro K, Van Cutsem E, SC O, Bodoky G, Shimada Y, et al. Ramucirumab plus paclitaxel versus placebo plus paclitaxel in patients with previously treated advanced gastric or gastrooesophageal junction adenocarcinoma (RAINBOW): a doubleblind, randomised phase 3 trial. Lancet Oncol. 2014;15:1224-35.

5. Garon EB, Ciuleanu TE, Arrieta O, Prabhash K, Syrigos KN, Goksel T. Ramucirumab plus docetaxel versus placebo plus docetaxel for second-line treatment of stage IV non-small-cell lung cancer after disease progression on platinum-based therapy (REVEL): a multicentre, double-blind, randomised phase 3 trial. Lancet. 2014;384(9944):665-73.

6. Mackey JR, Ramos-Vazquez M, Lipatov O, McCarthy N, Krasnozhon D, Semiglazov V. Primary results of ROSE/ TRIO-12, a randomized placebo-controlled phase III trial evaluating the addition of ramucirumab to first-line docetaxel chemotherapy in metastatic breast cancer. J Clin Oncol. 2015;33(2):141-8.
7. Tabernero J, Yoshino T, Cohn AL, Obermannova R, Bodoky G, Garcia-Carbonero R. Ramucirumab versus placebo in combination with second-line FOLFIRI in patients with metastatic colorectal carcinoma that progressed during or after first-line therapy with bevacizumab, oxaliplatin, and a fluoropyrimidine (RAISE): a randomised, double-blind, multicentre, phase 3 study. Lancet Oncol. 2015; 16(5):499-508.

8. Sashegyi A, Zhang Y, Lin Y, Binder P, Ferry D. Risk of gastrointestinal perforation in cancer patients receiving ramucirumab: a meta-analysis of randomized controlled trials. J Chemother. 2017;29(1):62-4.

9. Jemal A, Bray F, Center MM, Ferlay J, Ward E, Forman D. Global cancer statistics. CA Cancer J Clin. 2011:61:69-90.

10. Yoshikawa T, Rino Y, Yukawa N, Oshima T, Tsuburaya A, Masuda M. Neoadjuvant chemotherapy for gastric cancer in Japan: a standing position by comparing with adjuvant chemotherapy. Surg Today. 2014;44:11-21.

11. Badgwell BD, Camp ER, Feig B, Wolff RA, Eng C, Ellis LM. Management of bevacizumab-associated bowel perforation: a case series and review of the literature. Ann Oncol. 2008;19(3):577-82.

12. Burger RA, Brady MF, Bookman MA, Monk BJ, Walker JL, Homesley HD. Risk factors for $\mathrm{Gl}$ adverse events in a phase III randomized trial of bevacizumab in first-line therapy of advanced ovarian cancer: a Gynecologic Oncology Group Study. J Clin Oncol. 2014;32(12):1210-7.

13. Tanyi JL, McCann G, Hagemann AR, Coukos G, Rubin SC, Liao JB. Clinical predictors of bevacizumab-associated gastrointestinal perforation. Gynecol Oncol. 2011;120(3):464-9.

14. Yoshioka Y, Uehara K, Ebata T, Yokoyama Y, Mitsuma A, Ando Y, Nagino M. Postoperative complications following neoadjuvant bevacizumab treatment for advanced colorectal cancer. Surg Today. 2014;44:1300-6.

15. Sliesoraitis S, Tawfik B. Bevacizumab-induced bowel perforation. J Am Osteopath Assoc. 2011;111(7):437-41.

16. Haraoka S, Iwashita A. Metastatic tumor of the small intestine. Intestine. 2011;15(2):157-66.

17. Han ES, Monk BJ. What is the risk of bowel perforation associated with bevacizumab therapy in ovarian cancer? Gynecol Oncol. 2007;105(1):3-6.

18. Wang $Y$, Fei D, Vanderlaan M, Song A. Biological activity of bevacizumab, a humanized anti-VEGF antibody in vitro. Angiogenesis. 2004;7(4):335-45.

19. Choi Yl, Lee SH, Ahn BK, Baek SU, Park SJ, Kim YS. Intestinal perforation in colorectal cancers treated with bevacizumab (Avastin). Cancer Res Treat. 2008:40(1):33-5.

\section{Submit your manuscript to a SpringerOpen ${ }^{\circ}$ journal and benefit from:}

- Convenient online submission

- Rigorous peer review

- Open access: articles freely available online

- High visibility within the field

- Retaining the copyright to your article

Submit your next manuscript at springeropen.com 